\title{
Facteurs de production du lait et caractéristiques des fromages. II. Influence des caractéristiques des laits de troupeaux et des pratiques fromagères sur les caractéristiques du reblochon de Savoie fermier 1
}

\author{
B Martin *, JB Coulon
}

Laboratoire d'adaptation des herbivores aux milieux, INRA, 63122 Saint-Genès-Champanelle, France

(Reçu le 25 août 1994 ; accepté le 22 novembre 1994)

\begin{abstract}
Résumé - Les variations de la composition chimique et des caractéristiques sensorielles des fromages affinés ( $n=199$ ) ont été décrites à l'échelle de la quinzaine pendant 18 mois chez 6 producteurs de reblochon fermier de Haute-Savoie (France). Elles ont été mises en relation avec les caractéristiques des animaux (stade physiologique et variants génétiques des protéines du lait), les pratiques d'alimentation hivernales et estivales, les caractéristiques chimiques et rhéologiques des laits travaillés et la technologie mise en œuvre lors de la fabrication fromagère. La variabilité importante des caractéristiques chimiques et sensorielles des fromages réalisés par les producteurs a été faiblement liée directement aux caractéristiques des laits. Elle a en revanche été associée aux paramètres physiques du travail du lait en cuve traduisant l'intensité de l'égouttage. Lorsque les pratiques fromagères conduisent à une acidification très rapide ou au contraire très lente, la variabilité des caractéristiques des fromages est faible et leurs caractéristiques sensorielles témoignent d'un excès ou au contraire d'un manque d'égouttage. En revanche, lorsque l'acidification est moyenne, les caractéristiques des fromages sont plus variables et elles sont associées à la teneur des laits en urée, au stade physiologique des animaux, à la saison et, en été comme en hiver, à la nature des aliments distribués. En particulier, la nature des foins distribués en hiver et la composition floristique des pelouses d'alpage en été semblent jouer un rôle sur les caractéristiques des fromages affinés.
\end{abstract}

fromage fermier / pratique fromagère / lait de troupeau / alimentation / stade physiologique

Summary - Milk production and cheese characteristics. II. Influence of herd milk characteristics and cheesemaking methods on farmhouse French Reblochon cheese's characteristics. French Reblochon cheese chemical composition and sensory properties $(n=199)$ have been analysed every

1 Travail réalisé dans le cadre du programme de recherche-développement des Alpes-du-Nord.

*Adresse actuelle : GIS-SUACI Montagne, 11, rue Métropole, 73000 Chambéry, France. 
2 weeks over an 18-month-period on 6 dairy farms producing farmhouse cheese in the Haute-Savoie area (France). Variations were related to cheesemaking methods, chemical and rheological properties of herd milk used for cheesemaking, to cow characteristics (lactation stage and milk protein genetic variants) and feeding practices observed during the winter and summer seasons. Analyses showed an important variability of both chemical and sensory properties of farmhouse cheeses. Cheese characteristic variability is badly explained by only taking into account milk chemical composition or rheological properties. In fact, milk properties and cheesemaking methods are closely linked; this shows that farmers empirically modify the cheesemaking process according to chemical and rheological properties of milk, so that the cheese properties result from the association of one milk and one process. There are associations between milk characteristics and the cheesemaking process which lead to a slow or a rapid acidification: the variability of cheese characteristics is not important, and cheese properties result directly from the process. On the other hand, when acidification is average, the cheese property variability is important, and is associated with milk urea content, herd average lactation stage, season of the year, and in winter as in summer, to nature of the feeds (hay characteristics, floristic composition of pastures).

\section{farmhouse cheese / cheesemaking method / herd milk / feeding practice / lactation stage}

\section{INTRODUCTION}

Dans le domaine des produits laitiers et plus particulièrement des fromages, bien que l'influence de certains facteurs du milieu tels que les caractéristiques des animaux ou leur conduite alimentaire sur les caractéristiques des fromages affinés soit évoquée depuis longtemps par les fabricants fromagers, très peu de travaux les ont mis en évidence de façon objective (Urbach, 1990). Seuls les effets de la nature des aliments distribués aux animaux sur les caractéristiques sensorielles des laits ou des beurres sont bien connus (Urbach, 1990). En comparant des fromages d'origines différentes, quelques auteurs ont montré la plus grande richesse en composés aromatiques des fromages réalisés l'été et/ou en provenance des zones de montagne comparativement aux fromages d'hiver et/ou en provenance des zones de plaine (Dumont et Adda, 1978 ; Dumont et al, 1981). Berdagué et al (1990a et b) ont mis en évidence l'influence de certaines contraintes de production et de transformation du lait sur les caractéristiques chimiques et sensorielles des fromages affinés en comparant des emmentals classiques et "Grand-Cru". Par ailleurs, des travaux expérimentaux ont permis de mettre en évidence des différences de texture (Marziali et Ng-Kwai-Hang, 1986b) ou de goût (Mariani et al, 1976 ; Marie, 1991) liées au polymorphisme des protéines du lait. En revanche, très peu de travaux ont abordé l'influence des facteurs alimentaires sur les caractéristiques des fromages affinés (Garel et Coulon ,1990 ; Lightfield et al, 1993).

Devant la grande complexité des mécanismes de formation des caractéristiques sensorielles des fromages (Adda et al, 1982), la répercussion pratique, à l'échelle de l'exploitation ou de la fromagerie, des effets propres des différents facteurs mis en évidence en situation expérimentale a été très rarement abordée. Pourtant, à l'échelle de la fromagerie, les variations de la qualité des fromages affinés sont souvent difficilement interprétables (Chapman et Burnett, 1972 ; Grandison et al, 1985 ; Masson et al, 1981).

L'objectif de cet article est de décrire et d'analyser, chez 6 producteurs de reblochon fermier de la vallée de Thönes en Haute-Savoie, les variations des principales caractéristiques chimiques et sensorielles des fromages affinés en fonction des caractéristiques des animaux et de leur conduite 
(en particulier alimentaire), en tenant compte des paramètres technologiques mis en œuvre lors de la fabrication fromagère. Cet article fait suite à une première étude (Martin et Coulon, 1994) réalisée chez les mêmes producteurs, concernant les facteurs de variation de l'aptitude à la coagulation de laits de troupeaux. Quelques caractéristiques du reblochon sont précisées en annexe.

\section{MATÉRIEL ET MÉTHODES}

\section{Caractéristiques des exploitations}

Les 6 producteurs de reblochon fermier choisis pour cette étude sont tous localisés dans la vallée de Thônes en Haute-Savoie. Les principales caractéristiques des exploitations ont été décrites dans un article précédent (Martin et Coulon, 1995). Lors de la fabrication, le matériel utilisé et les principales techniques de fabrication fromagère ont été voisines d'une exploitation à l'autre: les principales opérations en cuve étaient réalisées manuellement (décaillage, brassage, soutirage, moulage), le caillé était moulé dans des moules individuels munis d'une toile perforee et les fromages étaient retournés à la main (en moule, au cours du pré-affinage ou en cave). En revanche, les modalités des différentes opérations ont différé sensiblement d'une exploitation à l'autre.

\section{Données recueillies}

Les données ont été recueillies au cours de passages bimensuels dans les 6 exploitations entre avril 1991 et octobre 1992. Elles concernent d'une part les caractéristiques des animaux, les pratiques d'alimentation et les caractéristiques des laits des cuves (cf Martin et Coulon, 1995) et d'autre part, les paramètres technologiques mis en œuvre par les producteurs lors de la fabrication et les caractéristiques chimiques et sensorielles des fromages affinés réalisés le jour du prélèvement de lait $(n=199)$.

Lors de chaque passage bimensuel sur les exploitations, les principaux paramètres tech- nologiques utilisés lors du travail du lait en cuve (durée des différentes phases, températures) ont été relevés. Les variables décrivant la maturation du lait (nature des levains, température, durée), la coagulation (température, dose de présure, temps de gélification et temps de coagulation total), les températures observées lors du découpage du caillé et du moulage, les durées de découpage, de brassage du mélange caillé-lactosérum, la durée totale comprise entre la fin du brassage et le moulage et la durée totale de fabrication ont été retenues pour la description des pratiques fromagères. La cinétique d'acidification des fromages réalisés les jours antérieurs $(\mathrm{pH}$ des fromages âgés de $12 \mathrm{~h}$, $24 \mathrm{~h}$ et de 7 jours) et la température du local de préaffinage et de la cave ont également été relevés.

\section{Fromages affinés}

\section{Identification et prélèvement}

Un fromage, choisi au milieu de la table d'égouttage, a été identifié lors de chaque suivi de fabrication. Ce fromage a subi les mêmes soins que l'ensemble des autres fromages de la fabrication jusqu'à l'âge de 15 jours environ où il a été emballé et acheminé au laboratoire pour finir son affinage dans des conditions identiques et contrôlées de température $\left(15^{\circ} \mathrm{C}\right)$ et d'hygrométrie (95\%).

\section{Composition chimique des fromages affinés}

Les analyses ont été réalisées sur une portion de fromage dont la croûte a été enlevée sur une épaisseur de $2 \mathrm{~mm}$ environ. Cet échantillon était ensuite broyé, homogénéisé et compactè dans un flacon. Le $\mathrm{pH}$ des fromages a été mesuré par introduction d'une électrode combinée à aiguille Ingold (Lot 406) dans le flacon de fromage broyé. La teneur en matière sèche des fromages a été déterminée par dessiccation à $103^{\circ} \mathrm{C}$ pendant $20 \mathrm{~h}$ de $5 \mathrm{~g}$ environ de fromage pesés exactement. Le dosage de la matière grasse des fromages a été réalisé par la méthode butyrométrique de Heis (1961) modifiée par Pien (1976). L'extrait sec dégraissé a été défini comme étant le complément à 100 de l'humidité du fromage dégraissé. 


\section{Analyses sensorielles}

Les analyses sensorielles ont été réalisées toutes les semaines par un jury d'experts composé de 5 à 8 personnes. Les membres du jury accordaient dans un premier temps une note d'appréciation globale à l'aspect extérieur du fromage (/5), à la pâte (ouverture/2, texture/3), au goût (/7) et à l'odeur (/3). Ils définissaient ensuite une liste de descripteurs caractérisant chacun des attributs du fromage. Le vocabulaire était libre ; il décrivait essentiellement les défauts du fromage, sans notation d'intensité. Au cours de l'ensemble des analyses sensorielles réalisées, 53 descripteurs ont été utilisés. Seuls les descripteurs utilisés dans plus de $3 \%$ des cas ont été retenus pour les analyses ultérieures. Ils sont au nombre de 37 qui caractérisent l'aspect extérieur $(n=14)$, la texture $(n=9)$, le goût $(n=8)$, l'ouverture de la pâte $(n=4)$ et l'odeur des fromages $(n=2)$.

\section{Traitement des données}

Les 199 fromages ayant fait l'objet des analyses sensorielles ont été classés en groupes homogènes à l'aide d'une classification ascendante hiérarchique $(\mathrm{CAH}$, logiciel $\mathrm{ADDAD})$ réalisée à partir des résultats d'une analyse factorielle des correspondances (AFC, logiciel ADDAD), dans laquelle les caractéristiques chimiques et sensorielles des fromages ont participé à la construction des axes. La classification des fromages a été réalisée à partir de leurs coordonnées sur les 8 premiers axes de l'AFC qui permettent de prendre en compte $50 \%$ de l'inertie totale. Une partition en 6 classes de fromages a été retenue.

De façon similaire, les pratiques fromagères recensées ont été regroupées en 6 classes homogènes à l'aide d'une $\mathrm{CAH}$ des coordonnées factorielles des fabrications sur les 4 premiers axes d'une AFC. Dans cette AFC, les variables décrivant la maturation du lait (nature des ferments, durée et température de maturation), la cinétique d'acidification et les modalités du travail en cuve ont été introduites en variables actives.

Dans un premier temps, le croisement des types de pratiques fromagères avec les types de fromages a permis de mettre en évidence un effet propre des conditions de fabrication sur les caractéristiques des fromages affinés. Ensuite, afin de s'affranchir de l'effet des pratiques fromagères sur les caractéristiques des fromages, les asso- ciations entre les caractéristiques des laits, les pratiques d'élevage et les caractéristiques des fromages ont été étudiées en ne considérant que des sous-ensembles de fromages pour lesquels les paramètres technologiques ont été semblables, c'est-à-dire en se plaçant intra-type de pratiques fromagères.

\section{RÉSULTATS}

\section{Caractéristiques des fromages}

\section{Moyennes et variabilité}

Les analyses chimiques et sensorielles ont été réalisées sur des fromages âgés de 25,4 jours en moyenne. Une variabilité importante a été relevée aussi bien pour leurs caractéristiques chimiques que sensorielles (tableau I). L'extrait sec total, le gras/sec et le $\mathrm{pH}$ des fromages affinés ont varié respectivement entre 43,8 et $60,2 \%$, entre 44,5 et $57,7 \%$ et entre 5,0 et 7,2 . Les notes de satisfaction globale ont varié entre 3 et 18/20. L'ensemble de l'échelle de notation a été utilisée pour les notes de texture, d'ouverture et d'odeur. Les notes de goüt et d'aspect ont varié respectivement entre 0 et $4,5 / 5$ et entre 0 et $6 / 7$. Les fréquences d'utilisation des principaux descripteurs des fromages sont relevées dans le tableau II.

\section{Typologie des fromages selon leurs caractéristiques (tableau III)}

Les fromages de type A se caractérisent par un extrait sec dégraissé élevé $(34,9 \%)$, un gras/sec et un $\mathrm{pH}$ faibles (respectivement $50,3 \%$ et 5,5 ). Ces fromages ont obtenu une note globale plutôt bonne $(11 / 20)$. Ils ont fréquemment présenté le défaut de "talon droit" $(54 \%)$, leur pâte a été trop "ferme" $(39 \%)$, elle a manqué de "fondant" $(19 \%)$ et a été fréquemment "farineuse" $(19 \%)$. Leur pâte se caractérise éga- 
Tableau I. Composition chimique et caractéristiques sensorielles des fromages affinés $(n=199)$. Chemical composition and sensory properties of ripened cheese $(n=199)$.

\begin{tabular}{lcccc}
\hline & Moyenne & Minimum & Maximum & Écart type \\
\hline Âge des fromages (jours) & 25,4 & 20 & 32 & 3,8 \\
Composition chimique & & & & \\
pH & 5,67 & 4,96 & 7,23 & 0,34 \\
Extrait sec total (\%) & 50,05 & 43,8 & 60,2 & 2,61 \\
Matières grasses (\%) & 25,48 & 21,5 & 57,7 & 2,35 \\
Extrait sec dégraissé (\%) & 33,0 & 27,5 & 43,6 & 2,4 \\
& & & & \\
Analyses sensorielles & & 3 & 18 & 2,7 \\
Note globale (/20) & 10,0 & 0 & 4,5 & 1,2 \\
Aspect (/5) & 2,2 & 0 & 3 & 0,8 \\
Pâte (/3) & 1,6 & 0 & 6 & 1,1 \\
Ouverture (/2) & 1,1 & 0 & 3 & 0,8 \\
Goût (/7) & 3,2 & 0 & & \\
Odeur (/3) & 1,8 & & & \\
& & & & \\
\hline
\end{tabular}

lement par sa très faible ouverture. Les défauts de goût les plus marqués ont été "alcoolisés/levurés» (42\%) et «acides» $(22 \%)$.

A l'opposé, les fromages de types $E$ et $F$ ont été caractérisés par un extrait sec dégraissé faible associé à un gras/sec et un $\mathrm{pH}$ plus élevés. Les fromages de type $\mathrm{E}$ ont obtenu une note globale de 12,9/20 essentiellement grâce à leur pâte, leur goût et leur odeur, très appréciés des notateurs. Leur pâte a été jugée le plus souvent «fondante" (14\%) et leur goût a été le plus souvent "neutre» mais n'a pas présenté de défauts majeurs. À l'opposé, les fromages de type $F$ ont obtenu les plus faibles notes globales essentiellement en raison de leur aspect extérieur et de leur odeur. Ils ont été excessivement «relâchés» et «déformés", leur croûtage a été "poisseux», et leur pâte a été "coulante». Ils ont par ailleurs été caractérisés par une odeur «désagréable» marquée, une ouverture excessivement "chargée" et "grosse», un goût "ammoniaqué» très fréquent (54\%).
L'extrait sec dégraissé des fromages des types B, C et D a été intermédiaire entre les 2 situations extrêmes précédentes. Leur gras/sec et leur $\mathrm{pH}$ ont été variables et ils ont obtenu des notes d'appréciation globale moyennes ou faibles. Les fromages des types B se caractérisent par une fréquence importante des défauts de pâte "ferme" $(26 \%)$ associée à la présence de "mucor" $(35 \%)$ et de "peau de crapaud" $(26 \%)$. Par ailleurs, leur goût a été jugé dans $32 \%$ des cas "alcoolisé/levuré" et les défauts d'ouverture de la pâte ont été très rares. Les fromages de type $C$ ont également fréquemment été caractérisés par le défaut de pâte "ferme" (30\%). Ils ont été plus humides et moins gras que ceux de type $B$ et ils ont obtenu une mauvaise note d'ouverture, jugée très souvent "aveugle» ou trop "grosse» et d'odeur, jugée "désagréable" dans $40 \%$ des cas. Les fromages de type $\mathrm{D}$ ont été caractérisés par une faible note d'appréciation globale $(8,0 / 20)$ essentiellement imputable à leurs faibles notes de texture, d'ouverture et de goût. Ils ont 
Tableau II . Fréquence d'apparition des descripteurs les plus importants (>3\%).

Most important sensory properties frequency.

Descripteur

Fréquence (\%)

Aspect

Talon droit 31

Talon irrégulier $\quad 10$

Relâché 6

Déformé 43

Creux 6

Cavités 16

Mucor 8

Trop blanc 11

Peau de crapaud 5

Fissuré/sec 26

Flore hétérogène $\quad 15$

Manque de flore 13

Poisseux 10

Goût

Acide 43

Salé $\quad 17$

Neutre 7

Alcoolisé $\quad 17$

Ecoeurant 8

Amer 13

Sucré/miel 16

Ammoniaqué 12

Pâte

Coulante 4

Fondante 3

Elastique 7

Collante 53

Farineuse 12

Plâtreuse 4

Ferme 22

Blanche

Hétérogène

Odeur

Désagréable

Cave

Ouverture

Excessive 16

Manque d'ouverture $\quad 17$

Trop grosse $\quad 37$

Irrégulière 12 présenté des défauts fréquents «d'ouverture excessive" $(51 \%)$ de la pâte associés à des goûts "acide" (68\%), "sucré" $(32 \%)$ et «écœurant» $(22 \%)$.

\section{Pratiques fromagères}

\section{Moyennes et variabilité (tableau IV)}

Les paramètres technologiques utilisés par les producteurs sont décrits dans le tableau IV. Une variabilité particulièrement importante a pu être observée aussi bien pour les conditions de maturation du lait (réalisée au cours de la traite) que pour les modalités du travail du lait en cuve.

\section{Typologie des pratiques fromagères (tableau V)}

La typologie des pratiques fromagères a permis de discriminer les paramètres technologiques essentiellement en fonction de la cinétique d'acidification, de la nature des levains, et de la durée de maturation.

Les pratiques fromagères de types 1 et 2 se caractérisent essentiellement par une acidification rapide ( $\mathrm{pH}$ voisin de 5,3 à $12 \mathrm{~h}$ ) et par une maturation longue réalisée avec des levains thermophiles constitués de cultures lyophilisées associées à des grands levains. Elles se différencient par les températures relevées au cours de la fabrication et par la durée de brassage du mélange caillé/lactosérum précédant le moulage, plus élevées pour les fabrications de type 1 que pour celles de type 2 .

À l'opposé, les pratiques fromagères de types 5 et 6 se caractérisent par une acidification très lente ( $\mathrm{pH}$ voisin de 5,7 à $12 \mathrm{~h}$ ) associée à une maturation courte et à l'utilisation exclusive de grands levains (yaourts du commerce). Ces 2 types de pratiques se différencient entre elles essentiellement par le $\mathrm{pH}$ des fromages âgés de $24 \mathrm{~h}$ qui 
Tableau III. Description des types de fromages obtenus par $\mathrm{CAH}$.

Cheese types description.

$\begin{array}{lllllllll}\text { Type de fromage } & A & B & C & D & E & F & E^{1}\end{array}$

Nombre de fromages

Âge (jours)

Composition chimique

$\begin{array}{llllll}59 & 31 & 30 & 37 & 29 & 13 \\ 25,0 & 26,3 & 26,6 & 24,9 & 24,2 & 25,8\end{array}$

$25,0 \quad 26,3 \quad 26,6 \quad 24,9 \quad 24,2 \quad 25,8$

3,7

ns

$\begin{array}{rrrrrrrr}5,5 & 5,8 & 5,4 & 5,7 & 5,8 & 6,0 & 0,3 & P<0,01 \\ 52,0 & 51,0 & 48,8 & 49,7 & 48,2 & 46,9 & 2,1 & P<0,01 \\ 50,3 & 51,4 & 50,1 & 51,3 & 51,6 & 51,2 & 2,3 & P<0,05 \\ 34,9 & 33,6 & 32,3 & 32,4 & 31,0 & 30,1 & 1,9 & P<0,01\end{array}$

Analyses sensorielles

Note globale (/20)

Aspect (/5)

Pâte (/3)

Ouverture (/2)

Goût (/7)

Odeur (/3)

$\begin{array}{rrrrrrrr}11,0 & 8,9 & 9,6 & 8,0 & 12,8 & 8,1 & 2,2 & P<0,01 \\ 2,7 & 1,3 & 2,5 & 2,3 & 2,9 & 1,2 & 1,0 & P<0,01 \\ 1,5 & 1,6 & 1,7 & 1,1 & 2,4 & 1,5 & 0,7 & P<0,01 \\ 1,4 & 1,5 & 0,6 & 0,8 & 1,2 & 1,0 & 0,6 & P<0,01 \\ 3,3 & 3,0 & 3,5 & 2,2 & 4,1 & 3,2 & 1,0 & P<0,01 \\ 2,1 & 1,5 & 1,3 & 1,6 & 2,2 & 1,2 & 0,8 & P<0,01\end{array}$

Principaux descripteurs (\%)

Aspect

Talon droit

Relâché

Poisseux

Mucor

Peau de crapaud

\section{Texture}

Pâte ferme

Manque de fondant

Coulant

Fondant

Collant

Pâte blanche

Goût

Acide

Salé

Neutre

Alcoolisé

Savon

Amer

Sucré

Ammoniaqué

\section{Ouverture}

$\begin{array}{lrrrrrrr}\text { Ouverture } & & 0 & 0 & 51 & 17 & 23 & P<0,01 \\ \text { Ouverture excessive } & 7 & 0 & 50 & 35 & 34 & 46 & P<0,01 \\ \text { Ouverture grosse } & 7 & 16 & 67 & 0 & 14 & 0 & P<0,01 \\ \text { Aveugle } & 19 & 6 & 47 & \\ \text { Odeur désagréable } & 7 & 10 & 40 & 16 & 14 & 38 & P<0,01\end{array}$

1 Écart type résiduel.

1 Residual standard deviation.

$\begin{array}{rrrrrrl}54 & 29 & 10 & 32 & 10 & 8 & P<0,01 \\ 5 & 3 & 3 & 0 & 10 & 23 & P<0,1 \\ 2 & 10 & 7 & 0 & 14 & 69 & P<0,01 \\ 7 & 35 & 3 & 0 & 0 & 8 & P<0,01 \\ 0 & 26 & 0 & 3 & 0 & 8 & P<0,01\end{array}$

$\begin{array}{rrrrrrl}39 & 26 & 30 & 8 & 0 & 8 & P<0,01 \\ 19 & 3 & 7 & 0 & 0 & 0 & P<0,05 \\ 0 & 0 & 7 & 0 & 0 & 38 & P<0,01 \\ 2 & 3 & 0 & 0 & 14 & 0 & P<0,01 \\ 56 & 65 & 67 & 49 & 45 & 23 & P<0,1 \\ 0 & 0 & 0 & 22 & 0 & 8 & P<0,01\end{array}$

$\begin{array}{rrrrrrr}42 & 29 & 43 & 68 & 38 & 23 & P<0,05 \\ 25 & 16 & 30 & 8 & 10 & 0 & P<0,05 \\ 7 & 3 & 3 & 3 & 24 & 8 & P<0,05 \\ 22 & 32 & 7 & 11 & 14 & 15 & P<0,1 \\ 5 & 13 & 3 & 22 & 0 & 0 & P<0,01 \\ 14 & 16 & 20 & 8 & 10 & 8 & \text { ns } \\ 14 & 13 & 10 & 32 & 14 & 8 & P<0,1 \\ 5 & 10 & 0 & 27 & 3 & 54 & P<0,01\end{array}$


Tableau IV. Paramètres technologiques moyens relevés entre avril 1991 et octobre $1992(n=199)$. Average cheese-making parameters $(n=199)$.

Moyenne Min Max Écart type

\section{Maturation}

Dose de levains mésophiles (I/1000 I) $(n=34)$

$\begin{array}{cccc}0,4 & 0,3 & 0,7 & 0,01 \\ 2,7 & 1,9 & 5,0 & 0,5 \\ 1,6 & 1,0 & 2,4 & 0,4 \\ i & 1 & 1 & 1 \\ 73,6 & 15 & 155 & 32 \\ 32,5 & 28 & 38 & 1,4\end{array}$

\section{Acidification}

$\mathrm{pH} 12 \mathrm{~h}$

$\mathrm{pH} 24 \mathrm{~h}$

$\mathrm{pH} 7$ jours

Travail en cuve

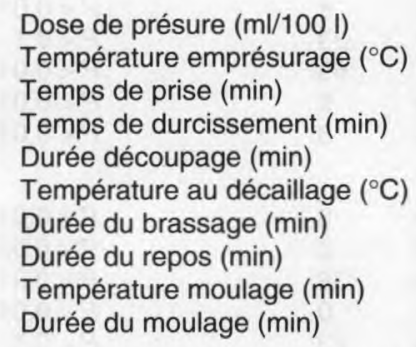

Matière grasse du lactosérum $(\mathrm{g} / \mathrm{l})$

\section{Affinage}

Température séchoir $\left({ }^{\circ} \mathrm{C}\right)$

Température cave $\left({ }^{\circ} \mathrm{C}\right)$

$\begin{array}{rrlr}25,1 & 14 & 45 & 4,4 \\ 33,3 & 31 & 36 & 1,0 \\ 16,5 & 8 & 28 & 3,8 \\ 33,0 & 10 & 83 & 11,0 \\ 8,3 & 2 & 22 & 3,9 \\ 32,2 & 26 & 35 & 1,3 \\ 5,0 & 0 & 25 & 4,7 \\ 4,5 & 0 & 18 & 2,8 \\ 32,8 & 28 & 37 & 1,4 \\ 14,3 & 5 & 30 & 5,1 \\ 8,3 & 4,0 & 16,8 & 1,97\end{array}$

est resté très élevé pour les pratiques de type 6 où le mélange caillé/lactosérum n'a pas été réchauffé et a été faiblement brassé avant le moulage.

Pour les pratiques fromagères de 3 et 4 , la cinétique d'acidification et la nature des levains thermophiles ont été intermédiaires entre les 2 situations précédentes. Le $\mathrm{pH}$ des fromages âgés de $12 \mathrm{~h}$ a été respectivement de 5,41 et 5,52 pour les pratiques fromagères de type 3 et 4 qui se différencient par la température du moulage et les durées des différentes opérations, plus élevées pour les fabrications de type 3 que pour celles de type 4 . 
Tableau V. Description des types de pratiques fromagères obtenues par $\mathrm{CAH}$. Cheese-making groups description.

$\begin{array}{lllllllllll}\text { Type de pratiques fromagères } & 1 & 2 & 3 & 4 & 5 & 6 & \text { ETR }^{1}\end{array}$

Nombre de fromages

$\begin{array}{llllll}32 & 16 & 35 & 51 & 48 & 19\end{array}$

\section{Maturation}

Thermophiles (effectifs)

Grands levains seuls

Lyophilisés seuls

Grands levains + lyophilisés

Durée (min)

Température $\left({ }^{\circ} \mathrm{C}\right)$

$\begin{array}{cccccccc}2 & 0 & 18 & 33 & 44 & 19 & & P<0,01 \\ 0 & 0 & 13 & 9 & 4 & 0 & & \\ 30 & 16 & 2 & 8 & 0 & 0 & & \\ 133 & 95 & 58 & 61 & 55 & 65 & 16 & P<0,01 \\ 31,9 & 32,0 & 33,2 & 33,3 & 31,5 & 33,2 & 1,2 & P<0,01\end{array}$

Acidification

$\begin{array}{llllllllc}\text { pH 12h } & 5,27 & 5,35 & 5,52 & 5,41 & 5,69 & 5,74 & 0,22 & P<0,01 \\ \text { pH 24 h } & 5,17 & 5,21 & 5,42 & 5,26 & 5,39 & 5,62 & 0,20 & P<0,01 \\ \text { pH 7 jours } & 5,12 & 5,10 & 5,11 & 5,09 & 5,11 & 5,16 & 0,12 & n s\end{array}$

Travail en cuve

Dose de présure $(\mathrm{ml} / 100 \mathrm{l})$

Température emprésurage $\left({ }^{\circ} \mathrm{C}\right)$

Temps de prise ( $\mathrm{min})$

Temps de durcissement ( $\mathrm{min}$ )

Durée décaillage ( $\mathrm{min}$ )

Température au décaillage $\left({ }^{\circ} \mathrm{C}\right)$

Durée du brassage (min)

Durée du repos (min)

Température moulage ( $\mathrm{min}$ )

$\begin{array}{rrrrrrrr}25,4 & 26,5 & 26,0 & 23,4 & 24,5 & 28,2 & 4,1 & P<0,01 \\ 34,1 & 32,6 & 34,4 & 33,0 & 32,6 & 33,1 & 0,7 & P<0,01 \\ 14,9 & 13,5 & 15,6 & 15,9 & 19,9 & 16,5 & 3,3 & P<0,01 \\ 22,1 & 37,4 & 42,1 & 33,4 & 30,0 & 39,3 & 9,3 & P<0,01 \\ 5,9 & 6,3 & 8,3 & 8,1 & 5,8 & 6,9 & 3,4 & P<0,01 \\ 33,0 & 32,1 & 33,3 & 32,5 & 30,8 & 31,9 & 1,0 & P<0,01 \\ 4,5 & 0,4 & 6,8 & 5,0 & 10,2 & 1,9 & 4,5 & P<0,01 \\ 3,0 & 3,0 & 6,9 & 3,8 & 5,2 & 4,0 & 2,5 & P<0,01 \\ 33,4 & 31,8 & 33,7 & 32,0 & 33,8 & 31,0 & 1,1 & P<0,01\end{array}$

Durée découpage-moulage ( $\mathrm{min}$ )

14

Durée totale du travail en cuve (min) 51

$\begin{array}{lllll}10 & 26 & 20 & 21 & 20 \\ 60 & 85 & 75 & 71 & 75\end{array}$

nt

nt

1 Écart type résiduel ; nt : non testé.

${ }^{1}$ Residual standard deviation; nt: not tested.

\section{Facteurs de variation}

\section{des caractéristiques des fromages}

\section{Ensemble des fromages}

Le croisement des types de fromages et des types de pratiques fromagères fait ressortir des associations marquées $(P<0,01)$ (tableau VI) qui permettent de mettre en évidence l'effet du type de pratiques fromagères d'une part sur les caractéristiques moyennes des fromages, mais surtout sur leur variabilité. Les pratiques fromagères assurant une acidification rapide (types 1 et 2) ont été à l'origine des fromages dont la variabilité a été la plus faible : 43 des 48 fromages obtenus se répartissent dans les 3 types de fromages caractérisés par leur extrait sec dégraissé élevé et par l'absence 
Tableau VI. Effet des pratiques fromagères sur les caractéristiques des fromages. Influence of cheese making methods on cheese characteristics.

Types de pratiques fromagères

Types de fromages

$\begin{array}{ccccccc} & A & B & C & D & E & F \\ \begin{array}{ccccc}1+2 \\ \text { (acidification rapide) }\end{array} & 18 & 11 & 14 & 1 & 4 & 0 \\ \begin{array}{c}3+4 \\ \text { (acidification moyenne) }\end{array} & 25 & 9 & 14 & 12 & 17 & 7 \\ \begin{array}{c}5+6 \\ \text { (acidification lente) }\end{array} & 16 & 11 & 2 & 24 & 8 & 6 \\ & & & & & & \end{array}$

de défauts d'ouverture excessive de la pâte (types A, B et C).

À l'opposé, les pratiques fromagères conduisant à une acidification lente (types 5 et 6) ont été à l'origine dans plus de la moitié des cas de fromages caractérisés par leur extrait sec dégraissé faible et par des défauts fréquents d'ouverture excessive de la pâte : les fromages de type $D$ et $F$ sont associés dans respectivement 65 et $45 \%$ des cas aux acidifications lentes.

Les pratiques fromagères pour lesquelles l'acidification a été moyenne (types 3 et 4) ont conduit globalement aux fromages dont la variabilité a été la plus importante : tous les types de fromages ont été observés.

Les pratiques fromagères ont cependant été fortement associées aux caractéristiques des laits (tableau VI). Les pratiques fromagères assurant un égouttage en cuve poussé (températures élevées et/ou brassage et repos importants) ont été appliquées aux laits pauvres en protéines ou provenant des troupeaux dont le stade de lactation était le plus avancé. C'est le cas notamment des pratiques de type 1 et 3 . À l'opposé, les pratiques fromagères caractérisées par un égouttage en cuve faible (températures et durées de brassage et de repos faibles) ont été associées aux laits les plus riches en protéines et provenant des troupeaux dont le stade de lactation était le plus faible (type 2). Ainsi, les fromagers ont adapté leurs pratiques fromagères aux aptitudes originelles des laits et les caractéristiques des fromages sont donc la résultante d'un type de pratiques fromagères appliqué à un type de lait.

\section{Fromages élaborés à technologie comparable : influence des pratiques d'élevage sur les caractéristiques des fromages}

Par la suite, seules les pratiques fromagères de types 3 et 4 ont été considérées. Dans ce cas, la variabilité des caractéristiques des fromages a été la plus importante et elle ne s'explique pas par la nature des levains, la cinétique d'acidification ou la qualité bactériologique des laits.

Caractéristiques des laits associées aux types de fromages (tableau VII)

Les gels les plus fermes ont été associés préférentiellement aux fromages de type A, dont l'extrait sec dégraissé a été le plus élevé bien que les différences n'aient pas été significatives (tableau VII). La proportion de protéines coagulables dans les pro- 
Tableau VII. Principales caractéristiques des laits associées aux types de pratiques fromagères. Associations between cheese making methods and milk and cheese characteristics.

\begin{tabular}{|c|c|c|c|c|c|c|c|c|}
\hline & \multicolumn{6}{|c|}{ Types de pratiques fromagères } & \multirow[b]{2}{*}{ ETR $^{1}$} & \\
\hline & 1 & 2 & 3 & 4 & 5 & 6 & & \\
\hline Flore totale $(\log / \mathrm{ml})^{2}$ & 6,53 & 6,29 & 6,19 & 6,21 & 6,05 & 6,01 & 0,39 & $P<0,01$ \\
\hline Flore coliforme (log/ml) & 1,95 & 1,25 & 1,57 & 1,61 & 2,30 & 2,47 & 0,70 & $P<0,01$ \\
\hline Taux protéique (g/kg) & 33,9 & 31,9 & 30,8 & 31,1 & 31,0 & 31,5 & 1,15 & $P<0,01$ \\
\hline Taux butyreux (g/kg) & 39,7 & 37,0 & 33,2 & 34,2 & 34,6 & 37,1 & 2,8 & $P<0,01$ \\
\hline Temps de gélification (min) & 11,9 & 12,4 & 14,7 & 13,1 & 14,0 & 13,8 & 1,7 & $P<0,01$ \\
\hline Fermeté du gel (mm) & 41,1 & 37,3 & 35,8 & 36,4 & 36,8 & 38,1 & 2,1 & $P<0,01$ \\
\hline Stade de lactation (jours) & 195 & 105 & 127 & 120 & 125 & 133 & 33 & $P<0,01$ \\
\hline Fin de lactation (\%) & 36 & 1 & 10 & 8 & 11 & 9 & 12 & $P<0,01$ \\
\hline
\end{tabular}

1 Écart type résiduel. 2 Lait additionné en levains.

1 Residual standard deviation. 2 Milk added with starters.

téines totales a été significativement plus faible $(P<0,05)$ pour les fromages de type $C$ et la teneur des laits en urée a été plus faible pour les fromages de type $\mathrm{E}(P<0,1)$.

Les principales différences entre types de fromages ont été observées pour les variables décrivant les caractéristiques des troupeaux. Les fromages de type $B$ et dans une moindre mesure ceux de type $A$ et $D$ (dont l'extrait sec a été le plus élevé) ont été associés préférentiellement aux cuves pour lesquelles la proportion de lait provenant des animaux en début de lactation ( $<60$ jours) a été la plus importante $(P<0,01)$ et où le pourcentage de vaches gravides a été le plus faible $(P<0,01)$.

\section{Pratiques d'élevage associées} aux types de fromages (tableau VIII)

À technologie comparable, les différents types de fromages ont été associés en premier lieu à la saison $(P<0,01)$ : les fromages des types $A$ et $C$ (défauts de pâte ferme fréquents) ont été observés seulement ou préférentiellement au cours de la période estivale alors que les fromages de type $F$ (extrait sec dégraissé très faible, aspect remouillé et goût ammoniaqué) ont été observés uniquement au cours de la période hivernale. Cet effet de la saison va ainsi globalement dans le sens d'un meilleur égouttage des caillés issus de laits d'été comparativement aux laits d'hiver. Cet effet ne s'explique pas par une acidification plus rapide des fromages d'été.

En hiver, comparativement aux fromages de type $E$ (meilleures notes globales essentiellement pour la pâte et le goût, et extrait sec dégraissé faible), les fromages de type A (défaut de pâte ferme et extrait sec dégraissé élevé) ont été associés à des quantités de foin distribuées plus faibles $(-3$ $\mathrm{kg} / \mathrm{j}, P<0,1)$ et à une moins bonne adaptation des quantités d'aliments concentrés au niveau de production des animaux. Néanmoins, les principales différences ont été observées au niveau de la nature des fourrages secs et des aliments concentrés distribués. Les plus importantes proportions de foin de Crau ont été associées aux fromages des types $A$ et $B$ comparativement aux fromages des types $E$ et $F$ bien que les différences n'aient pas été significatives. 
Tableau VIII. Caractéristiques des laits et pratiques d'élevage associées aux types de fromages pour les pratiques fromagères de types 3 et 4 .

Influence of milk characteristics and herd breeding conditions on cheese characteristics for cheese making groups 3 and 4.

Type de fromage

$\begin{array}{lllllll}A & B & C & D & E & F & E^{1}\end{array}$

Nombre

\section{Caractéristiques du lait}

Taux de leucocytes (x1000/ml)

Coliformes $(\mathrm{log} / \mathrm{ml})$

Flore totale $(\mathrm{log} / \mathrm{ml})^{2}$

Taux butyreux $(\mathrm{g} / \mathrm{kg})$

Taux protéique $(\mathrm{g} / \mathrm{kg})$

Protéines coagulables (\%TP)

Calcium total $(\mathrm{g} / \mathrm{kg})$

Urée ( $\mathrm{mg} / \mathrm{l}$ sérum)

$\mathrm{pH}$

Temps de gélification (min)

Fermeté du gel $(\mathrm{mm})$

Stade de lactation moyen (jours)

$\%$ d'animaux en début de lactation $(<60 j)$

$\%$ d'animaux en fin de gestation (\%)

$\%$ de caséine $\kappa \mathrm{B}$

$\%$ de $\beta$-lactoglobuline B

$\begin{array}{llllll}25 & 9 & 14 & 12 & 17 & 7\end{array}$

$\begin{array}{llllllll}103 & 66 & 129 & 92 & 91 & 71 & 64 & \text { ns }\end{array}$

$\begin{array}{llllllll}2,33 & 1,82 & 2,19 & 1,76 & 1,75 & 1,56 & 1,0 & \text { ns }\end{array}$

$\begin{array}{llllllll}6,14 & 6,15 & 6,14 & 6,19 & 6,35 & 6,25 & 0,39 & \text { ns }\end{array}$

$\begin{array}{llllllll}33,8 & 33,0 & 33,4 & 33,2 & 34,5 & 34,9 & 3,0 & \text { ns }\end{array}$

$\begin{array}{llllllll}31,2 & 30,3 & 31,2 & 30,6 & 31,0 & 31,1 & 0,96 & P<0,1\end{array}$

$\begin{array}{llllllll}79,1 & 79,6 & 78,6 & 79,2 & 79,0 & 79,6 & 0,73 & P<0,05\end{array}$

$\begin{array}{llllllll}1,19 & 1,16 & 1,20 & 1,19 & 1,20 & 1,17 & 0,04 & \text { ns }\end{array}$

$\begin{array}{llllllll}383 & 402 & 392 & 406 & 296 & 442 & 90 & P<0,1\end{array}$

$\begin{array}{llllllll}6,68 & 6,64 & 6,67 & 6,66 & 6,67 & 6,62 & 0,03 & P<0,01\end{array}$

$\begin{array}{llllllll}28,0 & 25,6 & 29,1 & 26,2 & 28,2 & 26,0 & 4,0 & \text { ns }\end{array}$

$\begin{array}{lllllll}36,7 & 35,1 & 36,5 & 35,8 & 36,2 & 35,3 & 1,7\end{array}$ ns

$\begin{array}{llllllll}121 & 99 & 131 & 122 & 131 & 121 & 27 & P<0,1\end{array}$

$\begin{array}{llllllll}33 & 41 & 20 & 35 & 21 & 24 & 15 & P<0,01\end{array}$

$\begin{array}{llllllll}47 & 40 & 61 & 42 & 58 & 51 & 14 & P<0,01\end{array}$

$\begin{array}{llllllll}52 & 53 & 53 & 47 & 48 & 49 & 6 & P<0,1\end{array}$

$\begin{array}{llllllll}48 & 46 & 48 & 51 & 49 & 50 & 7 & \text { ns }\end{array}$

Facteurs de production du lait

Saison

Hiver
Été

$\begin{array}{cccccc}7 & 4 & 0 & 5 & 8 & 6 \\ 18 & 5 & 14 & 7 & 9 & 1\end{array}$

$P<0,01$

Hiver

Quantité de foin ( $\mathrm{kg} / \mathrm{vache} / \mathrm{j})$

Foin de Crau (\%)

Foin de $2^{\mathrm{e}}$ cycle (\%)

Concentré distribué pour $25 \mathrm{~kg}$ de lait $(\mathrm{kg} / \mathrm{j})$

dont céréales $(\mathrm{kg} / \mathrm{j})$

$\begin{array}{cccccccc}10,8 & 14,4 & \text { I } & 13,2 & 14,7 & 13,2 & 2,5 & P<0,1 \\ 66 & 64 & \text { I } & 49 & 34 & 37 & 32 & \mathrm{~ns} \\ 18 & 44 & \text { I } & 44 & 52 & 39 & 21 & P<0,1 \\ 6,2 & 6,6 & \text { I } & 6,1 & 7,3 & 8,1 & 1,9 & \mathrm{~ns} \\ 0,6 & 1,0 & \text { I } & 1,0 & 0,9 & 2,0 & 1,7 & P<0,05\end{array}$

Été

Type de pelouse (effectifs)

Gras

Moyen et maigre

Quantités de foin ( $\mathrm{kg} / \mathrm{vache} / \mathrm{jour}$ )

Concentré distribué pour $25 \mathrm{~kg}$ de lait (kg/j)

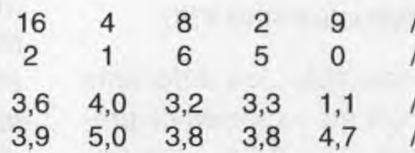

$P<0,01$

$2,5 \quad P<0,1$

1,3 ns

1 Écart type résiduel. 2 Lait ensemencé avec des levains.

1 Residual standard deviation. ${ }^{2}$ Milk added with starters. 
Par ailleurs, les proportions de foin de $2^{e}$ cycle et de foin de qualité moyenne ont représenté respectivement $52 \%$ et $0 \%$ du foin distribué pour les fromages de type $E$ contre respectivement 18 et $45 \%$ pour les fromages de type $\mathrm{A}$ qui ont également été associés à la distribution d'un seul type d'aliment concentré (concentré de production), à tous les animaux, quel que soit leur niveau de production.

Au cours de la période estivale, les différents types de fromages ont été associés principalement aux types de pelouses pâturées $(P<0,01)$ : les fromages de types A, B et $E$ ont été observés presque exclusivement sur les pelouses "grasses", alors que les fromages de types $C$ et $D$ ont été observés indifféremment ou préférentiellement sur les pelouses «moyennes" ou "maigres" $(P<0,01)$. Les plus faibles quantités de fourrages secs distribuées en plus du pâturage sont caractéristiques des fromages de type E. Par ailleurs, les fromages de type B ont été associés à des apports importants, à tous les animaux, d'aliments concentrés du commerce présentés sous forme agglomérée.

\section{DISCUSSION}

Comme l'ont mis en évidence Grandison et al (1985) au cours d'une étude réalisée chez des producteurs de fromages fermiers, cette étude permet dans un premier temps de montrer que ni les caractéristiques originelles des laits que nous avons mesurées, ni les paramètres technologiques utilisés par les producteurs, lorsque leurs effets sont abordés séparément ne permettent d'expliquer de façon satisfaisante les caractéristiques chimiques ou sensorielles des fromages. En pratique, les paramètres technologiques et les caractéristiques des laits des troupeaux ont été fortement associés. À l'exception des laits provenant des troupeaux dont le stade de lactation était le plus avancé, la technologie appliquée aux laits conduisant aux gels mous, comparativement aux laits conduisant aux gels fermes présentait les caractéristiques suivantes : 1) ils ont été travaillés à une température plus élevée ; 2 ) le découpage du caillé a été réalisé plus tôt, lorsque le caillé n'a pas encore atteint son degré de fermeté maximale ; 3 ) le brassage précédant le moulage a été plus long. Ces différentes modalités du travail du lait vont dans le sens d'un égouttage en cuve plus poussé (Weber, 1987) appliqué aux laits conduisant aux gels mous ou provenant des animaux en fin de lactation. Ces associations témoignent du savoir-faire des producteurs, qui se sont efforcés de compenser en partie les effets propres des caractéristiques des laits sur celles des fromages, qui peuvent être, à technologie identique, très importants (Martin et al, 1995).

Ces associations permettent aussi de confirmer indirectement que les laits conduisant aux gels les plus fermes possèdent une meilleure aptitude propre à l'égouttage et permettent d'obtenir des fromages dont l'extrait sec dégraíssé est plus élevé (Remeuf et al, 1991 ; Nuyts-Petit, 1991 ; Colin et al, 1992). II faut cependant distinguer les laits provenant des troupeaux dont le stade de lactation est le plus avancé qui, malgré leur comportement très favorable observé lors de la coagulation (gélification rapide et formation d'un gel ferme) ont été travaillés avec des paramètres technologiques plus favorables à l'égouttage en raison de leur égouttage difficile. Cet effet est donc bien connu des fromagers de cette région. De tels comportements des laits de fin de lactation n'ont pu être observés que dans des régions où les vêlages sont très groupés (Lucey et Fox, 1992). Ces auteurs ont attribué la mauvaise aptitude à l'égouttage des laits de fin de lactation d'une part à leur fort taux de leucocytes dont l'effet défavorable sur l'égouttage a été très clairement mis en évidence (Politis et Ng-KwaiHang et al, 1988 a, b et c) et d'autre part à 
leur teneur en plasmine élevée qui a pour effet principal de modifier la structure des micelles en altérant ainsi l'aptitude á la coagulation et à la synérèse des laits (Lucey et al, 1992). Dans notre cas, compte-tenu des faibles teneurs en leucocytes observées, il est plus vraisemblable de retenir Phypothèse d'une teneur élevée en plasmine qui, du fait du temps très court séparant la traite de la fabrication, n'aurait pas eu d'effet négatif sur l'aptitude à la coagulation du lait mais aurait par contre contribué à réduire l'aptitude à la synérèse au cours des stades ultérieurs de la fabrication fromagère. Enfin, la mauvaise aptitude à l'égouttage de ces laits pourrait aussi s'expliquer en partie par leur teneur élevée en protéines solubles, dont l'effet défavorable sur l'égouttage est parfois mentionné (Weber, 1987).

La variabilité importante des caractéristiques des fromages s'explique en premier lieu par la cinétique d'acidification observée lors des premières étapes de la réalisation du fromage. Les pratiques fromagères assurant une acidification rapide ont été à l'origine de fromages présentant globalement un extrait sec dégraissé élevé, une texture ferme et très peu d'ouvertures de la pâte. Ces caractéristiques témoignent d'un excès d'égouttage des fromages. Cela permet d'expliquer d'une part que la variabilité des caractéristiques des fromages ait été réduite, et d'autre part qu'on observe très rarement des fromages caractérisés par une fréquence importante d'ouverture de la pâte.

À l'opposé, les pratiques fromagères caractérisées par une cinétique d'acidification lente ont été associées principalement aux fromages dont la pâte a fréquemment présenté des défauts d'ouverture excessive, qui est restée blanche et qui a été caractérisée par un goût acide très fréquent. Ces défauts sont très caractéristiques d'un manque d'égouttage. La variabilité des caractéristiques des fromages observée pour ce type de pratiques fromagères s'explique essentiellement par la teneur initiale des laits en germes coliformes qui ont pour effet principal de provoquer des gonflements qualifiés de "précoces" et qui sont à l'origine des défauts d'ouverture excessive de la pâte.

Les pratiques fromagères caractérisées par une acidification intermédiaire ont été à l'origine des fromages dont les caractéristiques sensorielles ont été les plus variables. Cette variabilité n'a pas été associée à des cinétiques d'acidification ou des ferments de nature différente. Cela permet donc de montrer que dans ce cas, d'autres facteurs sont en cause pour expliquer la variabilité observée.

Cette variabilité a été très faiblement associèe aux caractéristiques des laits, et en particulier à leurs caractéristiques rhéologiques. Cette absence de liaison n'est pas surprenante compte-tenu de la faible variabilité observée lorsqu'on se place dans des conditions de fabrication fromagère comparables. Parmi les critères de la composition du lait mesurés, ce sont les fractions azotées mineures qui ont été les plus fortement associées aux caractéristiques sensorielles des fromages. En particulier, les plus fortes teneurs en urée ont été associèes aux fromages les moins appréciés et qui présentent globalement les plus importants défauts d'ouverture de la pâte. Des effets similaires de la teneur des laits en urée ont parfois été rapportés en fabrication de fromages de type Comté (Millet, 1988).

La variabilité des caractéristiques des fromages a été associée à la saison. En particulier, les fromages les plus fermes ont été obtenus en période estivale. Cette observation est certainement à rapprocher de l'effet favorable de la période de pâturage sur la fermeté du gel et sur l'égouttage des caillés (Martin et Coulon, 1995). Des effets marqués de la période de pâturage ont été souvent mis en évidence, d'une part 
sur les critères de la composition chimique des fromages (Chapman et Burnett, 1972 ; Masson et al, 1981 ; Collin et al, 1987 ; Guichard et al, 1987) et d'autre part sur leurs caractéristiques sensorielles (Dumont et Adda, 1978 ; Dumont et al, 1981 ; Berdagué et Grappin, 1988), la période de pâturage étant souvent associée aux fromages présentant la plus grande richesse aromatique. Dans notre étude, les analyses sensorielles des fromages permettant essentiellement de décrire les défauts des fromages, il n'a pas été possible de mettre en évidence des associations de ce type.

Pour chaque saison, les différents types de fromages obtenus ont été associés à la nature de l'alimentation des troupeaux : en hiver, à la nature des fourrages secs et des aliments concentrés et, en été, au type de pelouses pâturées et à la distribution de foin en complément du pâturage. Ces associations sont globalement en accord avec les observations empiriques réalisées par les fromagers de cette région qui constatent en particulier que l'introduction de foin de $2^{\mathrm{e}}$ cycle dans la ration en quantités importantes a pour effet principal d'assouplir la pâte des fromages, et que, au contraire, l'utilisation de fourrages secs au cours de la période estivale permet d'obtenir des fromages dont la pâte est plus ferme.

Des effets de la nature des pelouses ont également parfois été observés par les fromagers lors de changements de quartiers d'alpages. De tels effets de la nature des aliments sur les caractéristiques des fromages affinés ont cependant très rarement été mis en évidence par des travaux expérimentaux (Garel et Coulon, 1990 ; Lightfield et al, 1993). Dans notre cas, cet effet de la saison ou de la nature de l'alimentation des animaux ne s'explique pas par des différences de concentration des composés majeurs du lait (matières grasses, protéines) ou par son comportement lors de la coagulation. II est plus vraisemblablement lié à la composition de la matière grasse du lait pour laquelle il est bien établi que la saison et la nature de l'alimentation des animaux sont des facteurs de variation importants (Middaugh et al, 1988 ; Grummer, 1991 ; Lightfield et al, 1993). On sait en effet que la nature de l'alimentation des animaux peut jouer un rôle important sur les caractéristiques sensorielles du lait et du beurre (Urbach, 1990). D'autre part, des constituants mineurs du lait, issus directement des aliments offerts aux animaux, et en particulier de l'herbe pâturée, peuvent être à l'origine de différences de caractéristiques des fromages (Dumont et Adda, 1978).

\section{CONCLUSION}

Cette étude a permis de montrer que les variations de la qualité sensorielle des fromages sont soumises en premier lieu à la maîtrise de la technologie et plus particulièrement de la cinétique d'acidification au cours de la fabrication. Lorsque cette maîtrise est incomplète, les caractéristiques sensorielles des fromages résultent directement des défauts d'acidification. Ce n'est que lorsque la technologie est bien maîtrisée que les facteurs liés aux animaux ou aux conditions dans lesquelles ils sont conduits peuvent s'exprimer. Dans ce cas, les caractéristiques sensorielles des fromages ont été associées d'une part à la saison et d'autre part à la nature de l'alimentation des animaux.

L'originalité de cette étude, réalisée sous forme de suivis fins et de longue durée dans des exploitations produisant du fromage fermier, réside d'une part dans la variété des situations observées et d'autre part dans la prise en compte de l'ensemble des facteurs responsables de la qualité des fromages. Elle permet aussi, dans un domaine où un certain nombre de connaissances relève encore d'observations empiriques (Urbach, 1990), de mettre en évidence certains facteurs de variation (stade physiologique des 
animaux) et de proposer des hypothèses hiérarchisées pour d'autres (nature des aliments), y compris en ce qui concerne les interactions entre pratiques fromagères et pratiques d'élevage. Cependant, comptetenu de la démarche adoptée, certains de ces résultats relèvent plus de l'association entre des observations de nature différente qui peuvent être biaisées par des facteurs non contrôlés, que de la relation de cause à effet au sens strict. Les principales hypothèses émises devront donc faire l'objet d'une validation expérimentale et/ou de confrontations à d'autres situations pratiques (autres types de fromages, autres systèmes de production).

\section{REMERCIEMENTS}

Ce travail n'aurait pu être mené à bien sans la collaboration des producteurs de reblochon fermier de la vallée de Thônes chez qui cette étude a été réalisée (G Ballanfat, A Ménégon, S Suize et JP Tissot-Rosset et les membres du GAEC Bellachat et du GAEC de la Bottière) et sans le concours de E Combaz, et JM Barnabé, Y BurnetMerlin, B Mathieu et S Romand du service technique du Syndicat interprofessionnel du reblochon, de A Déchanet et G Jolivot du Syndicat de contrôle laitier de la Haute-Savoie, de I Philippot et X Folliet de la Chambre d'agriculture de la Haute-Savoie et F Farges de la coopérative des producteurs de reblochon fermier, qui ont largement contribué aussi bien à la mise en place qu'à la réalisation quotidienne de ce travail. Nous tenons à remercier tout particulièrement JF Chamba pour sa relecture critique de ce document, ainsi que toute son équipe de l'Institut technique du gruyère de La Roche-sur-Foron pour la réalisation des analyses sensorielles.

\section{ANNEXE}

Le reblochon de Savoie est un fromage à pâte pressée non cuite bénéficiant d'une appellation d'origine contrôlée. Sa production est soumise à une série de contraintes concernant à la fois les conditions de production et de transformation du lait.

Le lait doit provenir exclusivement d'une aire géographique correspondant aux 2/3 du département de la Haute-Savoie (France), il doit être travaillé à l'état cru et entier et sa production est limitée aux seules races Abondance, Montbeliarde ou Tarentaise nourries sans utilisation de fourrages fermentés. Le reblochon peut être fabriqué dans des fromageries artisanales ou industrielles à partir du mélange de laits de plusieurs troupeaux (reblochon «laitier" 9880 tonnes en 1993) ou à la ferme, 2 fois par jour, immédiatement après la traite, par le producteur lui-même, à partir du lait de son seul troupeau (reblochon «fermier»: 2972 tonnes en 1993).

\section{RÉFÉRENCES}

Adda J, Gripon JC, Vassal L (1982) The chemistry of flavour and texture generation in cheese. Food Chem 9, 115-129

Berdagué JL, Grappin R (1988) Affinage et qualité du gruyère de Comté. VI. Caractéristiques sensorielles des fromages. Lait 68, 189-204

Berdagué JL, Grappin R, Delacroix-Buchet A, Chaillet B (1990a) Caractérisation de l'emmental "grand-crun français. I. Composition physico-chimique. Lait 70,114

Berdagué JL, Grappin R, Chaillet B, Clément JF (1990b) Caractérisation de l'emmental *grand-cru" français. II. Analyses sensorielles. Lait 70, 133-145

Chapman HR, Burnett $J(1972)$ Seasonal changes in the physical properties of milk for cheesemaking. Dairy Ind 37, 207-211

Colin O, Laurent F, Vignon B (1992) Variations du rendement en pâte molle. Relation avec la composition chimique du lait et les paramètres de la coagulation. Lait 72, 307-319

Collin JC, Berdagué JL, Dognin-Bergeret M, Grappin R (1987) Affinage et qualité du gruyère de Comté. IV. Étude de la protéolyse. Lait 67, 299-318

Dumont JP, Adda J (1978) Occurence of sesquiterpenes in montain cheese volatiles. J Agric Food Chem 26 , 364-367

Dumont JP, Adda J, Rousseaux P (1981) Exemple de variation de l'arồme à l'intérieur d'un même type de 
fromage : le comté. Lebensm Wiss Technol 14, 198202

Garel JP, Coulon JB (1990) Effet de l'alimentation et de la race des vaches sur la fabrication de fromage d'Auvergne de Saint-Nectaire. INRA Prod Anim 3, 127-136

Grandison AS, Anderson M, Ford GD, Newell L (1985) Interrelationships between the diet fed to cows, composition and properties of milk and composition and quality of Cheshire cheese from farmhouse manufacturers. J Dairy Res 52, 587-593

Grummer RR (1991) Effect of feed on the composition of milk fat. J Dairy Sci 74, 3244-3257

Guichard E, Berdagué JL, Grappin R, Fournier N (1987) Affinage et qualité du gruyère de comté. $V$. Influence de l'affinage sur la teneur en composés volatils. Lait 67, 319-338

Heis $E$ (1961) Essai de dosage de la matière grasse dans le fromage par des méthodes rapides. Dtsch Molk Ztg 82, 67-70

Lightfield KD, Baer RJ, Schingoethe DJ, Kasperson KM, Brouk MJ (1993) Composition and flavor of milk and cheddar cheese higher in unsaturated fatty acids. J Dairy Sci 76, 1221-1232

Lucey JA, Fox PF (1992) Rennet coagulating properties of late-lactation milk: effect of $\mathrm{pH}$ adjustement, addition of $\mathrm{CaCl}_{2}$, variation in rennet level and blending with mid-lactation milk. Irish J Agric Food Res 31, 173-184

Lucey JA, Kindstedt PS, Fox PF (1992) Seasonality : its impact on the production of good quality Mozzarella cheese. In : 3rd Cheese Symposium, 28th29th October, Moorepark, Ireland

Mariani P, Losi G, Russo V, Castagnetti GB, Grazia L, Morini D, Fossa E (1976) Prove di caseificazione con latte caratterizzato dalle varianti A e B della kcaseina nella produzione del formaggio ParmigianoReggiano. Sci Tec Latt Casearia 27, 208-227

Marie C (1991) Influence du variant $C$ de la caséine $\beta$ sur la physico-chimie des laits de vache de race Tarine et sur leur aptitude à la transformation en fromages à pâte pressée cuite type Beaufort. Mémoire DEA. ENSA de Rennes

Martin B, Chamba JF, Coulon LB (1995) Effect of milk chemical composition and clotting ability on chemical and sensory properties of French reblochon cheeses made in laboratory and in farm (à paraître)
Martin B, Coulon JB (1995) Facteurs de production des laits et caractéristiques des fromages. I. Influence des facteurs de production sur l'aptitude à la coagulation des laits de troupeaux. Lait 75, 61-80

Marziali AS, Ng-Kwai-Hang KF (1986a) Relationships between milk protein polymorphisms and cheese yielding capacity. J Dairy Sci 69, 1193-1201

Marziali AS, Ng-Kwai-Hang KF (1986b) Effect of milk composition and genetics polymorphism on cheese composition. J Dairy Sci 69, 2533-2542

Masson C, Rousseaux P, Decaen C (1981) Variations géographiques et saisonnières de la qualité du fromage de Comté. Lait 61, 31-48

Middaugh RP, Baer RJ, Casper DP, Schingoethe DJ, Seas SW (1988) Characteristics of milk and butter from cows fed sunflower seeds. J Dairy Sci 71, 31793187

Millet J (1988) Incidence de la teneur en urée et de la standardisation du $\mathrm{pH}$ du lait sur la qualite d'un fromage à pâte pressée cuite : le Comté. Rev ENIL $131,7-14$

Nuyts-Petit V (1991) Influence des variants génétiques des caséines bovines sur l'aptitude fromagère du lait de vaches de races traditionnelles. Thèse de l'université de Compiègne

Pien J (1976) Détermination du taux de matière grasse des fromages. Techn Lait 878, 15-17

Politis I, Ng-Kwai-Hang KF (1988a) Effects of somatic cell counts and milk composition on the coagulating properties of milk. J Dairy Sci 71, 1740-1746

Politis I, Ng-Kwai-Hang KF (1988b) Association between somatic cell count of milk and cheese-yielding capacity. J Dairy Sci 71, 1720-1727

Politis I, Ng-Kwai-Hang KF (1988c) Effects of somatic cell count and milk composition on cheese composition and cheese making efficiency. J Dairy Sci 71, 17111719

Remeuf F, Cossin V, Dervin C, Lenoir J, Tomassone R (1991) Relations entre les paramètres physico-chimiques des laits et leur aptitude fromagère. Lait 71 . $397-421$

Urbach $\mathrm{G}$ (1990) Effects of feed on flavor in dairy foods. J Dairy Sci 73, 3639-3650

Weber F (1987) L'égouttage du coagulum. In : Le fromage (Eck A, ed). Lavoisier, Paris 